\title{
Application of Auto-ID in Agent-Based Manufacturing Control
}

\author{
T. N. Wong, C.B. Leung \\ Department of Industrial and Manufacturing Systems Engineering \\ The University of Hong Kong \\ Pokfulam Road, HONG KONG \\ Email: tnwong@hku.hk
}

\begin{abstract}
A feasibility study has been established to integrate agent and auto-ID technologies in manufacturing control applications. A multi-agent system (MAS) framework for intelligent manufacturing has been established. The intelligent MAS environment attempts to exploit the potential of Auto-ID (RFID in particular) technology in manufacturing applications. The aim is to evaluate the applications of Auto-ID, especially with RFID technology, in manufacturing control. This involves the establishment of the hardware and software interfaces to enable production and process data to be recorded and written in the Auto-ID devices. Experiments are being conducted to study the working requirements and parameters of the Auto-ID devices in the shopfloor environments. Subsequently, the RFID technology is adopted in a flexible assembly cell (FAC) to evaluate the feasibility of integrating the RFID devices in a multi-agent based manufacturing control system. A MAS infrastructure for FAC control has been developed to incorporate the coordination of the RFID devices.
\end{abstract}

Keywords-multi-agent system, Auto-ID, RFID, manufacturing control

\section{INTRODUCTION}

Conventional manufacturing systems are commonly established with the hierarchical control architecture. A central system controller is responsible for the major operation planning, control, and monitoring tasks for all components of the system. The hierarchical approach of centralized and sequential manufacturing planning, scheduling, and control mechanisms is deficient, in terms of flexibility and agility. Advanced manufacturing concepts such as agile manufacturing, web-based manufacturing and virtual enterprises demand a robust control software for the scheduling, planning and control functions. in supporting the modern production concepts.

Recently, researchers have identified the potential to apply multi-agent system (MAS) in solving various complex manufacturing system problems. The approach is concerned with the distribution and coordination of knowledge and actions in manufacturing environments involving a number of intelligent agents. A typical multi-agent manufacturing system is composed of autonomous components (machines, tools, parts, etc.) which communicate with other components and perform their functions without a central controller. MASs are designed to decentralize the control of the manufacturing systems, so as to reduce the complexity and to increase the flexibility of the systems. Such systems can also help to enhance fault tolerance.

Auto-ID devices, including barcodes and RFID (radio frequency identification) tags, have been used for automatic identification of products and items. In particular, the application of RFID technology in part identification has been attracting enormous attention in recent years.

This paper presents a feasibility study to integrate agent and auto-ID technologies in manufacturing control applications. In the study, a MAS framework for intelligent manufacturing control has been established for a flexible assembly cell. The intelligent MAS environment attempts to exploit the potential of RFID technology in manufacturing applications.

\section{AgEnT-BASED APPlications In MANUFACtURING CONTROL}

For the design of advanced manufacturing systems such as the flexible manufacturing system (FMS), researchers have recognised the importance to establish an intelligent and distributed control framework in the development of the control system to improve the flexibility and agility. Researchers have identified the potential of distributed AI (DAI) in solving various complex manufacturing system problems with MAS [1-3]. An MAS is a DAI system with a collection of autonomous agents. The agents in the MAS interact and collaborate to reach common objectives, while simultaneously each agent pursues its individual objectives.

Many researchers have attempted to develop agent-based architectures to support manufacturing activities. Since the late 1980s, a number of researchers have applied agent technology to perform production planning and control on the shopfloor level [4][5]. For MAS in manufacturing, it is natural to use autonomous agents to represent manufacturing resources such as products, parts, operations, workers, workstations, machines, tools, fixtures, or automated-guided vehicles to facilitate manufacturing resource planning, scheduling, and execution control [6].

From an enterprise's management point of view, Shen \& Norrie [6] discussed a number of motivations that justified the importance of intelligence and responsive behaviour of agentbased approach to manufacturing systems. For example, MetaMorph [7][8] was proposed as an adaptive agent-based 
architecture for concurrent engineering. Intelligent agents are established to pursue individual goals while satisfying constraints in combining the product design and production management issues. In the Autonomous Agents for the Rock Island Arsenal (AARIA) project, Parunak et al. [9] investigated large-scale resource allocation and system simulation using autonomous agents, in the context of factory scheduling. The project envisaged agent-oriented manufacturing entities (e.g. human users, machines, parts) interacting over a network as a manufacturing enterprise whose performance and functionality could exceed that of centrally controlled manufacturing systems. Wong et al. [10][11] have proposed a MAS-based decentralized negotiation for solving complicated integrated process planning and scheduling problems. The system is able to support dynamic process planning and rescheduling problem under unexpected disturbances [12].

For manufacturing control, multi-agents communicate and negotiate with each other to perform the operations based on the available local information. It includes the control of individual manufacturing resources or coordination of the recourses in shop-floor level [13]. Typically, agent models are built and implemented in order to attain the flexibility, reusability and fast response to both internal and external uncertainties in the shop floor environment [14-20]. Researchers have attempted to establish the intelligent and distributive manufacturing control structures as holonic manufacturing systems (HMS) [21].

Agent-based manufacturing control systems are mostly established in the hybrid structure as a compromise between the hierarchical and heterarchical control structures [18]. The main purpose of the agents is to obtain global objective from local agent solutions. The use of agents enriches the reliability and flexibility of planning and scheduling functions in the dynamic manufacturing system, and the agent structure provides reconfigurability to changes. The agents also provide the fault tolerance capability to the manufacturing environment. This capability is achieved by the re-allocation of the resources. For instance, Tang and Wong [18] proposed a reactive MAS for manufacturing system control. A prototype agent-based flexible manufacturing cell is established in which the agents can carry out simple task allocation via agent negotiation.

\section{RFID AND MANUFACTURING CONTROL}

A number of technologies have been developed and are being used in industry to achieve automatic identification: optical character recognition (OCR), magnetic strip, barcode, 2D barcode, radio frequency identification (RFID), biometrics, etc. Among these technologies, RFID has attracted the most attention in recent years. This has been motivated by the application of the RFID technology to track items through the supply chain. RFID is not a new technology; it has been used for many years [22]. In comparison with its main competitor the barcode, the main advantages of RFID are that it has more data storage capability, it does not require line of sight and RFID tags have unique codes.

An RFID system is made up of three components: RFID tag, RFID reader, and the RFID middleware. RFID tags are categorized as either passive or active. A passive tag does not possess a battery as its own voltage supply and it absorbs power from the RF field of the reader and reflects RF signal to the reader after adding information by modulating the received RF signal. In contrast, an active tag possesses its own power supply. An active tag can maintain data in RAM and it usually has a better read range th an passive tags and are suited to more applications. However, active tags are more expensive and they have limited operational lifetime due to power constraint. An RFID reader can read and write data received from RFID tags. It operates on a defined radio frequency according to a certain protocol.

RFID has been used in many areas such as postal tracking, document tracking, baggage, fare collection, library information systems, material handling, warehouse management, animal identification, waste management, and so on [23][24]. Nevertheless, most of the current research efforts in Auto-ID applications are on supply chain and logistics applications. Comparatively, there are only a relatively small number of research projects on RFID-based manufacturing applications [25][26]. Regarding manufacturing control, McFarlane et al. proposed to apply RFID technology and MAS to enhance the intelligence and performance of manufacturing control systems [27]. Frederic and Elgar [28] proposed an RFID-based real-time location system for enabling dispatching rules in a wafer fab. The RFID technology is implemented in the complex manufacturing process to capture shop floor information. In Zhou et al. [29], an RFID-based manufacturing data tracking system (RFID-MDTS) is designed to help users to collect all kinds of data and further track them in real time. In Wang and Lin [30], an agent-based manufacturing planning and control system has been developed with the integration of RFID and MAS.

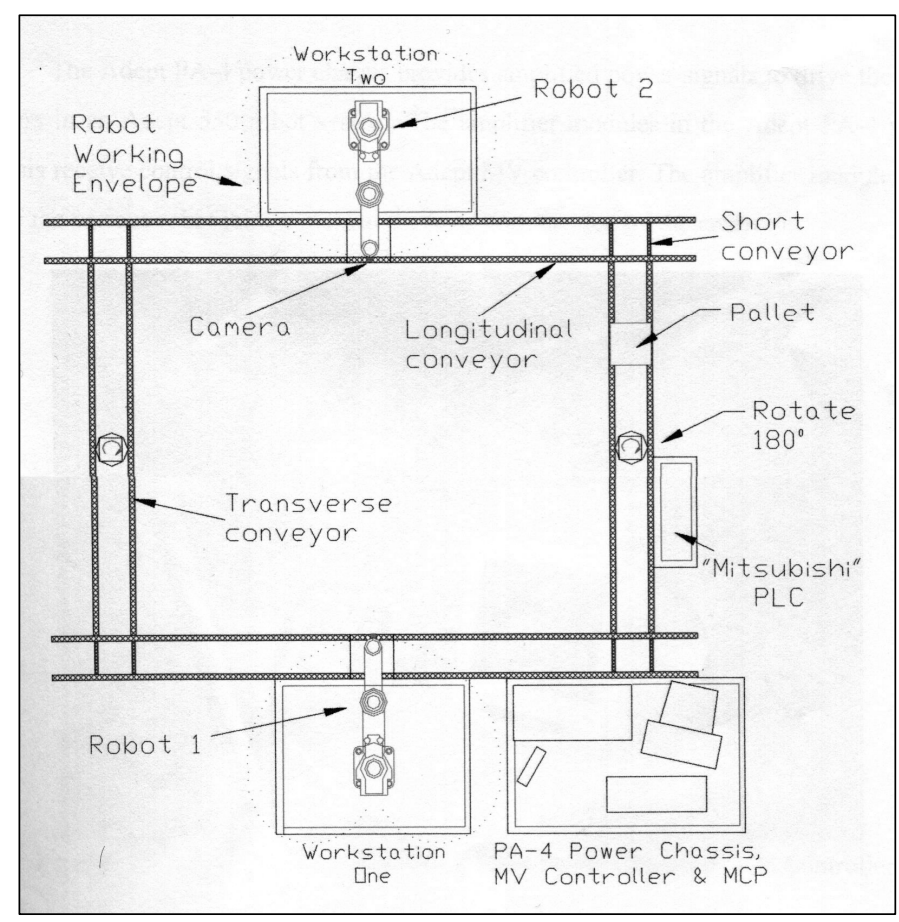

Figure 1. Configuration of the FAC 


\section{Configuration of The Assembly Cell}

Figure 1 depicts the configuration of a flexible assembly cell (FAC) [17]. The FAC comprises two Adept SCARAtyped assembly robots and a conveyor loop with 4 conveyors. The two robots are controlled by a single Adept programmable controller. Assembly operations and material handling tasks can be handled by these two robots. Stoppers are located at the front of the two robots (the area is called the robot processing area) so that pallets are clamped for robot processing. The robot controller is equipped with two serial communication ports for communication.

There are four conveyors in the FAC. They form a loop connection for pallets transportation. Sensors, pneumatic cylinders, signal indicators are controlled by a programmable logic controller (PLC). The PLC is employed as the central processing unit for the motion control of the conveyor system. Ladder diagram is used to prepare the control program for the control logics of the transportation system. The PLC is equipped with a serial communication port for communication.

\section{MAS FRAMEWORK}

A hybrid MAS framework for manufacturing planning and control activities is proposed. It consists of local agents, a supervisory agent, and a distributed agent platform. The local agents include AutoID agent, Robot Control agent, Conveyor agent, etc., representing different elements anf functions in the FAC. All the planning and control decisions are made via a negotiation-based approach amongst the local agents. The Supervisory Agent is designed to coordinate the negotiation and monitor the local decisions made by the local agents. The agent platform mainly includes an agent management system, a message transport protocol, and a database. The agent management system controls the lifecycle of all the agents in the framework. The message transport protocol provides message passing mechanism between the agents. The database records the information flow and decisions made by the agents. Figure 2 depicts some of the agents in the control system. In this example, five agents (scheduling agent, pallet identity agent, AutoID agent, conveyor agent and robot agent) are used to form the core working elements in the system.

\section{A. Agents and Agent Structure}

The MAS consists of agent units corresponding to specific functionalities in the physical configuration of the FAC. The Order-Handling agent provides the interface for the manufacturing system connecting with the external orderrequesting agent through the Internet communication or mobile communication media The Scheduling agent is responsible for coordinating the tasks between the pallet identity agent, the AutoID agent and the conveyor control agent. It links up with the central database to retrieve and save the overall system information. It is embedded with a specific scheduling algorithm to cater for the scheduled instruction in the FAC. The AutoID agent is responsible for the provision of the part information from the RFID tag. It decodes the RFID tagged data from the RFID readers and then extracts the appropriate part ID information and process data. This information is sent to the scheduling agent, the pallet identity agent and the conveyor agent. The Pallet Identity agent is responsible for the provision of the local pallet information and pallet position information from the sensor signal interpreter agent. The Conveyor Control agent is responsible for the provision of the local solution from the two sub-level conveyor agents. The information is required by the scheduling agent, the pallet identity agent and the AutoID agent. The Robot Control agent is responsible for the provision of the local robot manipulation. The agent also assigns the required control program to the

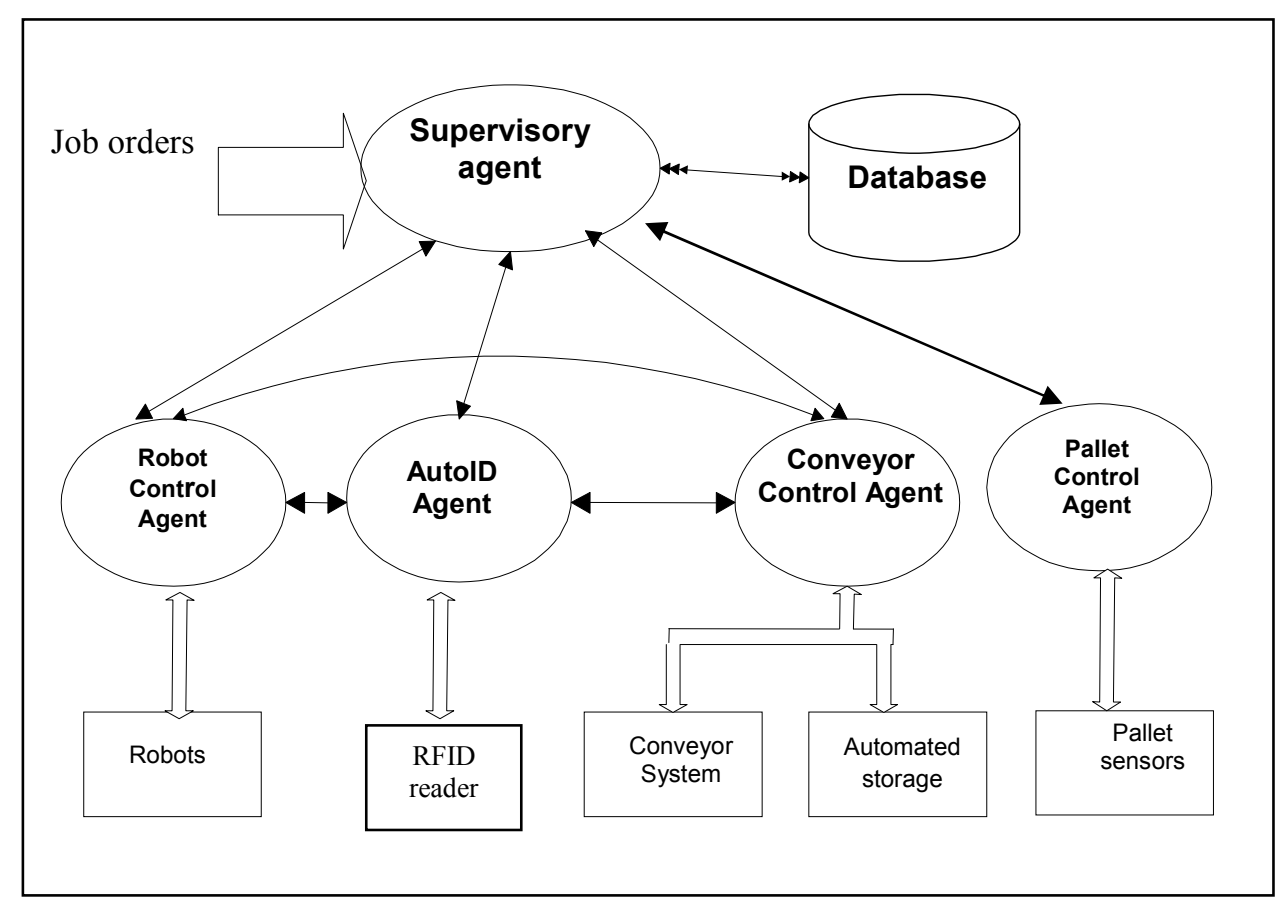

Figure 2. Architecture of the multi-agent system 


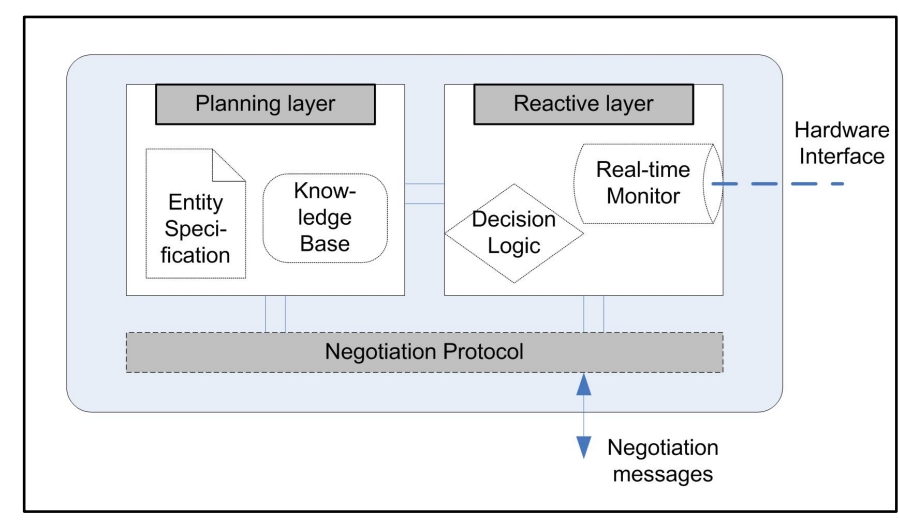

Figure 3. Hybrid layered structure of an agent

robot to meet the order requirements. The Sensor Signal Interpreter agent is responsible for the provision of the local sensor information from the sensor position translation agent. It co-operates with the pallet identity agent to identify the pallet position. The Sensor Position Translational agent translates the raw signal into the pallets' positional readable format to the controller.

As shown in Figure 3, agents in the proposed MAS are established with a hybrid structure of two layers: a planning and a reactive control layer. The proposed agent structure is a combination of reactive (behaviour-based) and belief-based agents. Purely behaviour-based agents have quick response, but there is a lack of reasoning power for planning and execution functions. On the other hand, if purely belief-based agents are used, the agent will have reasoning power but the system will be slower in response and weaker in robustness. As defined in Tang and Wong [18], this hybrid structure embraces the properties of the two distinctive types of agents in a hybrid layered approach.

The hybrid two-layer structure is a generic form of agents in the proposed MAS. Its reactive layer may be equivalent to the operational unit. This layer controls the hardware in the cell (AutoID, robot and conveyor line, etc.) according to the decisions from the planning layer. The planning layer acts as the decision unit. It decides the appropriate actions during the manufacturing process. It makes decision by considering the part information and machine status and consulting its knowledge base which is the database in the planning layer. Moreover, both planning and reactive layers have their own communication abilities. In most cases, an agent contains the third layer of underlying hardware which is a physical entity that the agent controls. For instance, the hardware is a RFID reader for the AutoID agent, or robot for the Robot Control agent. The reactive layer sends control signals to the hardware and gets feedback signal.

The reactive layer is responsible for the real-time operations of the agent. It employs the subsumption architecture and contains behaviours that couple stimuli and reactions directly. The layer is simple, has high effectiveness, and quick response. Its primary role is to ensure the execution of the plans and schedules. Due to the dynamic nature of the manufacturing system, the reactive layer provides real-time monitoring to the environments and to the hardware being connected. In the proposed generic structure, the reactive layer of one agent can send messages to the reactive layer of another agent. It greatly enhances the social ability of agents. It allows direct interactions between a number of reactive layers during realtime operation. Whenever a manufacturing disturbance occurs which may affect the original plans, it provides quick and realtime response to restore the plans and hence ensure the stability of the execution.

The planning layer possesses all necessary reasoning power for schedule searching. It is designed to perform task allocation within the manufacturing systems. It contains specific information which states the agent's objectives and its own status. A knowledge base exists to provide historic information and decisions which aid the agent to make negotiation decisions internally. From primitive requirement data to a set of plans or schedules, the planning layer possesses all necessary reasoning power for making good negotiation decisions. During the planning stage, it communicates with other agents for planning via message passing. The planning layer also monitors and regulates decided schedules. It makes adjustments of schedules during the execution stage.The planning layer and the reactive layer can communicate via messages. During the execution stage, the planning layer can send commands to the reactive layer and gets feedback. The planning and reactive layers are linked to each other so that the decisions and modifications can be updated instantly. They are both connected to the negotiation protocol which allows them to react and negotiate with other agents. Accordingly, complex control problems may be broken down into simpler problems that can be handled individually by a single reactive layer. The planning layer will act as a coordinator of a number of reactive layers to solve control problems.

\section{IMPLEMENTATION ISSUES}

The local agents, the supervisory agent, and the negotiation protocol are all implemented in the Java Agent Development environment JADE platform. It is a wellestablished platform for development agent applications and it is conformed to the FIPA (Foundation of Intelligent Physical Agent) standards. In JADE, user-defined agents are situated at agent containers. Containers may be in one or several computers that JADE are installed. Agents can communicate with others by passing agent-communication language (ACL) messages among or within containers. JADE is multi-platform and can be extended easily. Agents in the proposed system, including the Supervisory agent, AutoID agent, and Robot Control agent, can be easily extended from the basic agent class.

In the FAC, passive and read/write RFIG tags are used. In preliminary tests, the tags are used for recording the part identity and process information. For simplicity, the common 96-bit RFID tags are being used in the current implementation. The first 16 bits are used for header and tag ID and the subsequent 16 bits provide the part identity. That is, a total of 64 bits can be used for representing manufacturing process information including operation sequence and manufacturing status.

In operation, the AutoID agent scans the signal from the 
RFID readers by polling. When a pallet passes through the scanning envelop of the a reader, it will scan the RFID tag on the pallet. The recorded information will be sent to the AutoID agent and the Pallet Identity agent. To start processing in the FAC, the loaded pallets are put on the conveyor system. The pallet identity agent interprets the pallet identity numbers according to the pallet entry priorities. If a palleted part is identified to be the next part to be operated according to the task assignment sequence, the pallet identity agent will validate the pallet identity with information read by the AutoID agent. Corresponding to the code recorded in the RFID tag, the appropriate task information including the robot program can be retrieved from the database. The scheduling agent will send requests to the robot control agent and the conveyor control agent respectively. Receiving the pallet parking request from the scheduling agent, the conveyor control agent will direct the conveyor controller to position and clamp the pallet at the desired robot processing area. The robot control agent will then receive the robot processing request from the scheduling agent and hence it will send the corresponding robot program to the robot controller. The corresponding robot can then start the required assembly operations. After the robot has completed the assembly operation on the component according to the robot program, the robot controller will send the robot program end signal to the robot control agent. The ending acknowledgement signal will then be sent from the robot control agent to the scheduling agent, pallet identity agent and the conveyor control agent. The pallet will then be released from the robot processing area and put back onto the conveyor. The AutoID agent will then update the status of the part. The updated status is to be recorded in the database and the RFID tag. Finally, the conveyor will be restarted to transfer the pallets around the FAC.

\section{CONCLUDING REMARK}

A MAS infrastructure for manufacturing control has been developed to incorporate the RFID technology. In the MAS, there are various categories of agents, for example, AutoID agent, Supervisor agent, Robot Control agents and so on. In the manufacturing system, the RFID tags provide the information on the part identity, process information and operation status. The RFID reader scans a RFID tag in real-time operation and sends the tagged data to the AutoID agent. The proposed approach has demonstrated the advantage of applying AutoID devices such as RFID in agent-based manufacturing control as accurate and unique product and process information about each individual part can be otained during the manucturing processes.

\section{REFERENCES}

[1] J.S. Basran, et al., "Flexible agent-based robotic assembly cell" In: Proceedings of the 1997 IEEE International conference on Robotics and Automation, pp.3461-3466, 1997.

[2] A. Nagata and J. Hirai, "Distributed planning for assembly tasks by multiple manipulators". In: Proceedings of the IEEE International conference on Robotics and Automation, pp. 3522-3529, 1994.

[3] S. Franklin and A. Grasser, "Is it an agent, or just a program?" In: Proceedings of the third international workshop on Agent theories, architectures and languages Springer-Verlag, pp. 54-66, 1996.

[4] M.J. Shaw, "A distributed scheduling method for computer integrated manufacturing: the use of local area networks in cellular systems" International Journal of Production Research, 25(9), pp. 1285-1303, 1987.

[5] A.D. Baker, "Complete manufacturing control using a contract net: a simulation study" In: Proceedings of the International Conference of Computer Integrated Manufacturing, 1988, pp.100-109.

[6] W. Shen \& D.H. Norrie, "Agent-based systems for intelligent manufacturing: a state-of-the-art survey." International Journal of Knowledge and Information Systems, Vol.1, No.2, pp.129-156, 1999.

[7] S. Balasubramanian, et al. "Multi-agent planning and coordination for distributed concurrent engineering." International Journal of Cooperative Information Systems, Vol. 5, No.2-3, pp.53-179, 1996.

[8] W. Shen, et al., "MetaMorph II: an agent-based architecture for distributed intelligent design and manufacturing." Journal of Intelligent Manufacturing, Vol. 11, No.3, pp.237-251, 2000.

[9] H.V.D. Parunak et al., "The AARIA agent architecture: From manufacturing requirement to agent-based system design." Integrated Computer-Aided Engineering, Vol.8, No.1, pp.45-58, 2001.

[10] T.N. Wong et al., "An agent-based negotiation approach to integrate process planning and scheduling." International Journal of Production Research, Vol. 44, No.7, pp.1331-1351, 2006.

[11]T.N. Wong et al., "Dynamic shopfloor scheduling in multi-agent manufacturing systems." Expert Systems with Applications, Vol. 31, No. 3, pp.486-494, 2006.

[12]T.N. Wong et al., "Integrated process planning and scheduling/rescheduling with an agent-based approach." International Journal of Production Research, 44(18-19), pp.3627-3655, 2006.

[13] P. Leital, "Agent-based distributed manufacturing control: A state-of-the-art survey". Engineering Applications of Artificial Intelligence, vol 22, pp.979-991, 2009.

[14]H. Friedrich et al. "Integrating skills into multi-agents systems". Journal of Intelligent Manufacturing, Vol. 9, pp. 119-127, 1998. 
[15] S.K. Tso, et al. "A framework for developing an agentbased collaborative service-support system in a manufacturing information network". Engineering Applications of Artificial Intelligence, vol 12, pp. 43-57, 1999.

[16]D. McFarlane and S. Bussmann, "Developments in holonic production planning and control". International Journal of Production Planning and Control, Vol.11, No. 6, 522-536, 2000.

[17]C.K. Fan and T.N. Wong, 2003. "Agent-based architecture for manufacturing system control". Integrated Manufacturing Systems, Vol. 14, No. 7, 2003, pp 599-609.

[18]H.P. Tang and T.N. Wong, "Reactive multi-agent system for assembly cell control." Robotics and ComputerIntegrated Manufacturing, Vol. 21, No.2, pp.87-98, 2005.

[19]L. Monostori, et al. "Agent-based systems for manufacturing". Annals of the CIRP, vol. 55, No.2, pp.697-720, 2006.

[20] V. Marik and J. Lazansky, "Industrial applications of agents technologies". Control Engineering Practice, Vol.15, pp.1364-1380, 2007.

[21]R. Babiceanu and F. Chen, "Development and applications of holonic manufacturing systems: a survey". Journal of Intelligent Manufacturing, vol. 17, pp.111-131, 2006.

[22] Wikipedia, http://en.wikipedia.org/wiki/RFID (last accessed on 29 March 2010).

[23]D. McFarlane and Y. Sheffi, "The impact of automatic identification on supply chain operations". International Journal of Logistics Management, Vol. 14, No. 1, pp. 1$17,2003$.
[24] S.F. Wamba and Y. Bendavid, "Understanding the Impact of Emerging Technologies on Process Optimization: The Case of RFID Technology", Auto-ID White Paper. Retrieved Sept 2008, from http://autoidlabs.org/publications/page.html, 2008.

[25]N. Chokshi, et al., "Routes for integrating auto-ID systems into manufacturing control middlware environments". White Paper CAM-AUTOID-WH026, Auto-ID Centre, Institute for Manufacturing, University of Cambridge, UK., 2003.

[26] A. Garcia, et al. . "An agent-oriented design methodology for RFID improved manufacturing control". In: Proceedings of IEEE Internatinoal Conference on Emerging Technologies and Factory Automation, Prague, pp. 790-796. , 2006.

[27]D. McFarlane, et al.. "Auto ID systems and intelligent manufacturing control". Engineering Applications of Artificial Intelligence, Vol. 16, No.4, pp. 365-376, 2003.

[28] T. Frederic and F. Elgar, F. "On the value of location information to lot scheduling in complex manufacturing processes". International Journal Production Economics, Vol. 112, pp. 532-547, 2008.

[29] G. Zhou, et al. "Design of an RFID-based manufacturing data tracking system in plant production" in: Intelligent Robotics and Applications, First International Conference, ICIRA 2008, Wuhan, China, Oct 15-17, 2008, Part II, pp.688-696, 2008

[30]L.C. Wang and S.K. Lin. "A multi-agent based agile manufacturing planning and control system". Computers \& Industrial Engineering, Vol. 57, pp. 620-640. , 2009. 